The Hausdorff dimension of some random invariant graphs

Moss, $A$ and Walkden, C. P.

2011

MIMS EPrint: 2011.38

Manchester Institute for Mathematical Sciences

School of Mathematics

The University of Manchester

\footnotetext{
Reports available from: http://eprints.maths.manchester.ac.uk/

And by contacting: The MIMS Secretary

School of Mathematics

The University of Manchester

Manchester, M13 9PL, UK
} 


\title{
The Hausdorff dimension of some random invariant graphs
}

\author{
A. Moss ${ }^{* \dagger}$ and C.P. Walkden*
}

$3^{\text {rd }}$ May, 2011

\begin{abstract}
Weierstrass' example of an everywhere continuous but nowhere differentiable function is given by $w(x)=\sum_{n=0}^{\infty} \lambda^{n} \cos 2 \pi b^{n} x$ where $\lambda \in(0,1), b \geq 2, \lambda b>1$. There is a well-known and widely accepted, but as yet unproven, formula for the Hausdorff dimension of the graph of $w$. Hunt $[\mathrm{H}]$ proved that this formula holds almost surely on the addition of a random phase shift. The graphs of Weierstrass-type functions appear as repellers for a certain class of dynamical system; in this note we prove formulae analogous to those for random phase shifts of $w(x)$ but in a dynamic context. Let $T: S^{1} \rightarrow S^{1}$ be a uniformly expanding map of the circle. Let $\lambda: S^{1} \rightarrow(0,1), p: S^{1} \rightarrow \mathbb{R}$ and define the function $w(x)=\sum_{n=0}^{\infty} \lambda(x) \lambda(T(x)) \cdots \lambda\left(T^{n-1}(x)\right) p\left(T^{n}(x)\right)$. The graph of $w$ is a repelling invariant set for the skew-product transformation $T(x, y)=\left(T(x), \lambda(x)^{-1}(y-p(x))\right)$ on $S^{1} \times \mathbb{R}$ and is continuous but typically nowhere differentiable. With the addition of a random phase shift in $p$, and under suitable hypotheses including a partial hyperbolicity assumption on the skew-product, we prove an almost sure formula for the Hausdorff dimension of the graph of $w$ using a generalisation of techniques from $[\mathrm{H}]$ coupled with thermodynamic formalism.
\end{abstract}

\section{$\S 1 \quad$ Introduction}

The study of everywhere continuous but nowhere differentiable functions has a long history. The first, and perhaps most studied, example is the Weierstrass function

$$
w(x)=\sum_{n=0}^{\infty} \lambda^{n} \cos 2 \pi b^{n} x, \lambda \in(0,1), b \in \mathbb{N} .
$$

${ }^{*}$ School of Mathematics, The University of Manchester, Oxford Road, Manchester, M13 9PL, U.K.

${ }^{\dagger}$ A preliminary version of the results over this paper appeared in the first author's $\mathrm{PhD}$ thesis. Financially supported by EPSRC.

2010 Mathematics Subject Classification: 37C45, 37D20, 37H99. 
This series converges uniformly, hence $w$ is continuous. If $\lambda b>1$ then $w$ is nowhere differentiable.

More generally, consider the graph of an arbitrary function $w:[0,1] \rightarrow$ $\mathbb{R}:$

$$
\operatorname{graph}(w)=\{(x, w(x)) \mid x \in[0,1]\} \subset \mathbb{R}^{2} .
$$

If $w$ is differentiable then $\operatorname{graph}(w)$ is a 1-dimensional manifold and consequently has Hausdorff dimension 1. If $w$ is nowhere differentiable then $\operatorname{graph}(w)$ is typically a fractal and the dimension of $\operatorname{graph}(w)$ gives an indication of how irregular $w$ is.

Computing the box dimension of graph $(w)$ is often straightforward. Indeed, for the Weierstrass function $w$ one can easily check [BU] that

$$
\operatorname{dim}_{B} \operatorname{graph}(w)=2-\frac{\log \lambda^{-1}}{\log b} .
$$

It is widely conjectured that the Hausdorff dimension $\operatorname{dim}_{H} \operatorname{graph}(w)$ of the Weierstrass function is also given by (2).

There are examples [PU] of functions of the form (1) where $\cos 2 \pi b^{n} x$ is replaced by the $n$th Rademacher function (note that this is piecewise constant but not continuous) and $\lambda$ is a Pisot number for which $\operatorname{dim}_{H} \operatorname{graph}(w)<$ $\operatorname{dim}_{B} \operatorname{graph}(w)$. More generally, given any integers $n>m>1$, letting $\alpha=\log m / \log n$ and choosing any $s \in(1,2-\alpha)$, one can construct [PU, M] a Hölder continuous function $w_{s, \alpha}$ of exponent $\alpha$ such that $\operatorname{dim}_{B}\left(\operatorname{graph}\left(w_{s, \alpha}\right)\right)=$ $2-\alpha$ but $\operatorname{dim}_{H}\left(\operatorname{graph}\left(w_{s, \alpha}\right)\right)=s<2-\alpha$.

It is often the case, however, that if one introduces a random parameter into the construction of a fractal, then the conjectured value of the Hausdorff dimension for the non-random case can be proved to hold for almost every value of this parameter. Hunt proved in $[\mathrm{H}]$ that if $\theta=\left(\vartheta_{n}\right)_{n=0}^{\infty}$, where $\vartheta_{n} \in[0,1]$ are chosen uniformly and independently, then with

$$
w_{\theta}(x)=\sum_{n=0}^{\infty} \lambda^{n} \cos 2 \pi\left(b^{n}+\vartheta_{n}\right), \lambda \in(0,1), b \in \mathbb{N}, b \geq 2, \lambda b>1
$$

the Hausdorff dimension of $\operatorname{graph}\left(w_{\theta}\right)$ is

$$
\operatorname{dim}_{H} \operatorname{graph}\left(w_{\theta}\right)=2-\frac{\log \lambda^{-1}}{\log b} \text { a.s. }
$$

Indeed, as is remarked in $[\mathrm{H}]$, one can replace cos in (3) with a suitably smooth periodic function $p$ satisfying a mild condition on its critical points.

One can view $\operatorname{graph}(w)$ as the invariant set (indeed, a repeller) for a certain skew-product dynamical system. In order to make some of the objects below continuous, it is technically more convenient to work on the circle $S^{1}=\mathbb{R} / \mathbb{Z}$. Define $T: S^{1} \rightarrow S^{1}$ by $T(x)=b x \bmod 1$ and define

$$
\hat{T}: S^{1} \times \mathbb{R} \rightarrow S^{1} \times \mathbb{R}:(x, y) \mapsto\left(T(x), \lambda^{-1}(y-\cos 2 \pi x)\right) .
$$


Let $w$ be defined as in (1). Then $\hat{T}(\operatorname{graph}(w))=\operatorname{graph}(w)$. More generally, one can consider skew-products defined on $S^{1} \times \mathbb{R}$ of the form $\hat{T}(x, y)=$ $\left(T(x), \lambda(x)^{-1}(y-p(x))\right.$ where $p: S^{1} \rightarrow \mathbb{R}$ and $\lambda: S^{1} \rightarrow(0,1)$. Define

$$
w(x)=\sum_{n=0}^{\infty} \lambda(x) \lambda(T(x)) \cdots \lambda\left(T^{n-1}(x)\right) p\left(T^{n}(x)\right) .
$$

Then $\operatorname{graph}(w)$ is a $\hat{T}$-invariant repeller for $\hat{T}$.

One can then consider the case when $T$ is replaced by a uniformly expanding map of the circle. In the context of hyperbolic dynamics, one can often recognise the dimension of an invariant set as the solution of a certain equation, often called Bowen's equation, of the form $P(s f+g)$ where $P$ denotes the topological pressure. For many dynamically-defined fractal sets one can also often recognise the dimension as the entropy of the underlying dynamics divided by the Lyapunov exponent, with respect to an appropriate invariant measure.

In $[\mathrm{Be}]$ the box dimension of the graph of a function of the form (4) is calculated to be the unique solution $s$ to the equation $P\left((1-s) \log T^{\prime}+\right.$ $\log \lambda)=0$.

In this paper, we study the Hausdorff dimension of equations of the form (4) where the function $p$ is modified by the addition of a random phase shift. One aim is to put the results of $[\mathrm{H}]$ into the context of thermodynamic formalism. Indeed, we prove:

\section{Theorem 1.1}

Suppose that $T$ is a $C^{2}$ uniformly expanding map of the circle. Let $\lambda: S^{1} \rightarrow$ $(0,1)$ be $C^{1}$, and let $p: S^{1} \rightarrow \mathbb{R}$ be, for example, a polynomial or a finite sum of trigonometric functions. Define

$$
w_{\theta}(x)=\sum_{n=0}^{\infty} \lambda(x) \lambda(T(x)) \cdots \lambda\left(T^{n-1}(x)\right) p\left(T^{n}(x)+\vartheta_{n}\right)
$$

where the $\vartheta_{n}$ are chosen uniformly and independently from $S^{1}$. Then there exists a $T$-invariant probability measure $\mu_{0}$ such that

$$
\operatorname{dim}_{H} \operatorname{graph}\left(w_{\theta}\right)=1+\frac{h_{\mu_{0}}(T)+\int \log \lambda d \mu_{0}}{\int \log T^{\prime} d \mu_{0}} \text { a.s. }
$$

where $h_{\mu_{0}}(T)$ denotes the measure-theoretic entropy of $T$ with respect to $\mu_{0}$.

The precise statement and hypotheses are given below in Theorem 2.4. In particular, $\mu_{0}$ can be identified to be the equilibrium state of a certain Hölder continuous potential. We also make precise the conditions assumed on $p$. 
In $\S 2$ we give the necessary background and state two results which give an upper and lower bound, respectively, on $\operatorname{dim}_{H}\left(\operatorname{graph}\left(w_{\theta}\right)\right)$. In $\S 3$ we prove the upper bound. In $\S 4$, we prove the lower bound; the key estimate in $\S 4$ is a generalisation of the method used in $[\mathrm{H}]$.

\section{$\S 2$ Preliminaries and statement of results}

\section{$\S 2.1 \quad$ Expanding circle maps}

Let $T: S^{1} \rightarrow S^{1}$ be a $C^{1+\varepsilon}$ map, i.e. $T$ is continuous, continuously differentiable and the derivative is Hölder continuous. By replacing $T$ with $T^{2}$ if necessary, there is no loss in assuming that $T$ is orientation-preserving. There exists a partition of $S^{1}$ into intervals $I_{j}=\left[a_{j-1}, a_{j}\right], 1 \leq j \leq N$, (where the intervals are taken mod 1 if necessary) such that the restriction $T: I_{j} \rightarrow S^{1}$ is a homeomorphism on $I_{j}$ and a diffeomorphism on the interior of $I_{j}$. We assume that $T$ is uniformly expanding, in the sense that there exists $\beta>1$ such that $T^{\prime}(x) \geq \beta$ for all $x \in S^{1}$.

Let $T_{j}: S^{1} \rightarrow I_{j}, 1 \leq j \leq N$, denote the inverse branches of $T$. Let $x_{0}, x_{1}, \ldots, x_{n-1} \in\{1, \ldots, N\}$. Define

$$
\left[x_{0}, x_{1}, \ldots, x_{n-1}\right]=T_{x_{0}} T_{x_{1}} \cdots T_{x_{n-1}}\left(S^{1}\right)
$$

and note that this is an interval. We call such sets cylinders of rank $n$. Let $\mathcal{C}_{n}$ denote the set of all cylinders of rank $n$ and note that, for each $n \geq 1$, $\mathcal{C}_{n}$ is a partition of $S^{1}$.

\section{$\S 2.2$ Pressure}

Let $g: S^{1} \rightarrow \mathbb{R}$ be Hölder continuous. Define $|g|_{\alpha}=\sup _{x \neq y} \mid g(x)-$ $g(y) \mid / d(x, y)^{\alpha}$. There are many equivalent ways of defining the pressure $P(g)$ of $g$, and here we briefly review those that we will need in what follows.

\section{$\S$ 2.2.1 Pressure via cylinders}

Let $g: S^{1} \rightarrow \mathbb{R}$ be Hölder continuous. Define the pressure of $g$ to be

$$
P(g)=\lim _{n \rightarrow \infty} \frac{1}{n} \log \sum_{\left[x_{0}, x_{1}, \ldots, x_{n-1}\right] \in \mathcal{C}_{n}} \exp \sum_{j=0}^{n-1} g\left(T^{j}(x)\right)
$$

where the $x$ in the summand is taken to be any point in $\left[x_{0}, x_{1}, \ldots, x_{n-1}\right]$. It follows immediately from the definition and the following lemma that $P(g)$ is independent of the choice of points $x \in\left[x_{0}, x_{1}, \ldots, x_{n-1}\right]$. 


\section{Lemma 2.1}

Let $g: S^{1} \rightarrow \mathbb{R}$ be Hölder continuous of exponent $\alpha$. If $x, y$ are in the same cylinder of rank $n$ then

$$
\left|\sum_{j=0}^{n-1} g\left(T^{j}(x)\right)-g\left(T^{j}(y)\right)\right| \leq|g|_{\alpha} \frac{1}{1-\beta^{-\alpha}} .
$$

Proof. Recall that $T_{j}: S^{1} \rightarrow I_{j}$ denote the inverse branches of $T$. As $\left[x_{0}, x_{1}, \ldots, x_{n-1}\right]=T_{x_{0}} \circ T_{x_{1}} \circ \cdots \circ T_{x_{n-1}}\left(S^{1}\right)$ it follows that $\operatorname{diam}\left[x_{0}, x_{1}, \ldots, x_{n-1}\right] \leq$ $1 / \beta^{n}$. A straightforward calculation shows that if $x, y \in\left[x_{0}, x_{1}, \ldots, x_{n-1}\right]$ then

$$
\left|\sum_{j=0}^{n-1} g\left(T^{j}(x)\right)-g\left(T^{j}(y)\right)\right| \leq|g|_{\alpha} \sum_{j=0}^{n-1} d\left(T^{j}(x), T^{j}(y)\right)^{\alpha} \leq|g|_{\alpha} \frac{1}{1-\beta^{-\alpha}} .
$$

\section{$\S 2.2 .2$ Pressure via spanning sets}

We can also define pressure via spanning sets [W, for example]. Let $d(x, y)=$ $\min \{|x-y|, 1-|x-y|\}$ denote the metric on $S^{1}$ inherited from $[0,1]$ with 0 identified with 1 . Define a family of metrics $d_{n}$ by $d_{n}(x, y)=$ $\max \left\{d\left(T^{j}(x), T^{j}(y)\right) \mid 0 \leq j \leq n-1\right\}$. For $\delta>0$ and $x \in S^{1}$ define the $(n, \delta)$-Bowen ball $B_{n, \delta}(x)=\left\{y \in S^{1} \mid d_{n}(x, y)<\delta\right\}$. A subset $F \subset S^{1}$ is said to be $(n, \delta)$-spanning if $\bigcup_{x \in F} B_{n, \delta}(x)=S^{1}$.

Let $g: S^{1} \rightarrow \mathbb{R}$ be continuous. Define

$$
P_{n}(g, \delta)=\inf \sum_{x \in F} \exp \sum_{j=0}^{n-1} g\left(T^{j}(x)\right)
$$

where the infimum is taken over all $(n, \delta)$-spanning sets.

Define

$$
P(g, \delta)=\limsup _{n \rightarrow \infty} \frac{1}{n} \log P_{n}(g, \delta) .
$$

Then one can show that if $\delta_{1}<\delta_{2}$ then $P\left(g, \delta_{1}\right) \geq P\left(g, \delta_{2}\right)$ so that $P(g)=$ $\lim _{\delta \rightarrow 0} P(g, \delta)$ exists.

Recall that a dynamical system $T$ is said to be expansive with expansivity constant $\delta_{0}>0$ if $d\left(T^{n}(x), T^{n}(y)\right) \leq \delta_{0}$ for all $n \geq 0$ implies $x=y$. If $T$ is a uniformly expanding map of $S^{1}$ then $T$ is expansive with expansivity constant $1 / \beta$. One can show [W, Theorem 9.6] that if $\delta_{0}$ is an expansivity constant then $P(g)=P(g, \delta)$ whenever $\delta<\delta_{0} / 4$. 


\section{§2.2.3 Pressure via the variational principle}

We can also define $P(g)$ via the variational principle [W]. Let $g: S^{1} \rightarrow \mathbb{R}$ be continuous. Then

$$
P(g)=\sup \left\{h_{\mu}(T)+\int g d \mu\right\}
$$

where $h_{\mu}(T)$ denotes the entropy of $T$ and the supremum is taken over all $T$-invariant probability measures $\mu$.

For each Hölder continuous $g: S^{1} \rightarrow \mathbb{R}$ there exists a unique $T$-invariant probability measure $\mu_{g}$ which attains the supremum in (7), i.e. $P(g)=$ $h_{\mu_{g}}(T)+\int g d \mu_{g}$. We call $\mu_{g}$ the equilibrium state with potential $g$.

Equilibrium states have the following Gibbs property. Let $g: S^{1} \rightarrow \mathbb{R}$ be Hölder continuous and let $\delta>0$. Then there exists a constant $C(g, \delta)>1$ such that for any $x \in S^{1}$ and any Bowen ball $B_{n, \delta}(x)$ we have

$$
\frac{1}{C(g, \delta)} \leq \frac{\mu_{g}\left(B_{n, \delta}(x)\right)}{\exp \sum_{j=0}^{n-1} g\left(T^{j}(x)\right)-n P(g)} \leq C(g, \delta) .
$$

\section{$\S$ 2.2.4 Properties of pressure}

Let $f, g$ be Hölder continuous. It is clear from any of the definitions of pressure that if $f \leq g$ then $P(f) \leq P(g)$. In particular if $f \geq 0$ and $g$ is any function then $s \mapsto P(s f+g)$ and $s \mapsto P(-s f+g)$ are increasing and decreasing functions of $s$, respectively. It is also well-known that the dependence of $P(f)$ is continuous [W, for example] (indeed, analytic) on $f$, so that $s \mapsto P(s f+g), P(-s f+g)$ are continuous functions of $s$.

It is also clear from (7) that if $c$ is a constant then $P(g+c)=P(g)+c$ and that, if $c \geq 1$, then $P(c g) \leq c P(g)$.

\section{$\S 2.3 \quad$ Hausdorff dimension}

Let $E \subset \mathbb{R}^{n}$ and $s \geq 0$. The $s$-dimensional Hausdorff measure of $E$ is defined by

$$
\mathcal{H}^{s}(E)=\lim _{\delta \rightarrow 0} \inf \sum_{j} \operatorname{diam}\left(I_{j}\right)^{s}
$$

where the infimum is taken over all countable open covers $I_{j}$ such that $E \subset \bigcup_{j} I_{j}$ and the diameter diam $I_{j} \leq \delta$. The Hausdorff dimension of $E$ is defined by

$$
\operatorname{dim}_{H} E=\inf \left\{s \mid \mathcal{H}^{s}(E)=0\right\}=\sup \left\{s \mid \mathcal{H}^{s}(E)=\infty\right\}
$$

One can also characterise Hausdorff dimension in terms of energy integrals. Let $\mu$ be a probability measure supported on $E$. For $s \geq 0$ define the $s$-energy of $\mu$ to be

$$
I_{s}(\mu)=\iint \frac{d \mu(x) d \mu(y)}{\|x-y\|^{s}}
$$


and define the correlation dimension of $\mu$ to be $\sup \left\{s \mid I_{s}(\mu)<\infty\right\}$. Then the Hausdorff dimension $\operatorname{dim}_{H}(E)$ is the supremum of the correlation dimensions over all probability measures supported on $E$.

\section{$\S 2.4$ Random dynamical systems}

Let $\lambda: S^{1} \rightarrow(0,1)$ be Hölder continuous. Let $p: S^{1} \rightarrow \mathbb{R}$ be continuous. Let $T: S^{1} \rightarrow S^{1}$ be a uniformly expanding map of $S^{1}$. Define

$$
\hat{T}: S^{1} \times \mathbb{R} \rightarrow S^{1} \times \mathbb{R}:(x, y) \mapsto\left(T(x), \lambda(x)^{-1}(y-p(x))\right) .
$$

We introduce a random phase-shift as follows. Equip $S^{1}$ with Lebesgue measure. Let $\Omega=\left\{\left(\vartheta_{j}\right)_{j=0}^{\infty} \mid \vartheta_{j} \in S^{1}\right\}$. Equip $\Omega$ with the measure given by the Cartesian product of Lebesgue measure; we will denote this measure by $d \theta$.

Let $\tau: \Omega \rightarrow \Omega$ be the left shift map, so that if $\theta=\left(\vartheta_{j}\right)_{j=0}^{\infty}$ then $(\tau(\theta))_{j}=$ $\vartheta_{j+1}$. Define the random dynamical system $\tilde{T}$ by

$\tilde{T}: \Sigma \times \mathbb{R} \times \Omega \rightarrow \Sigma \times \mathbb{R} \times \Omega:(x, y, \theta)=\left(T(x), \lambda(x)^{-1}\left(y-p\left(x+\vartheta_{0}\right), \tau(\theta)\right)\right)$

and consider the projection onto the $(x, y)$ co-ordinates

$$
\hat{T}_{\theta}(x, y)=\left(T(x), \lambda(x)^{-1}\left(y-p\left(x+\vartheta_{0}\right)\right)\right) .
$$

Define $\lambda^{n}(x)=\lambda(x) \lambda(T(x)) \cdots \lambda\left(T^{n-1}(x)\right), \lambda^{0}(x)=1$. Then for each $\theta=\left(\vartheta_{j}\right)_{j=0}^{\infty} \in \Omega$, the function

$$
w_{\theta}(x)=\sum_{n=0}^{\infty} \lambda^{n}(x) p\left(T^{n}(x)+\vartheta_{n}\right)
$$

is a continuous function (as the sum converges uniformly), and $\operatorname{graph}\left(w_{\theta}\right)$ is $\hat{T}_{\theta}$-invariant. To see this, simply observe that

$$
\begin{aligned}
\hat{T}_{\theta}\left(x, w_{\theta}(x)\right) & =\left(T(x), \lambda^{-1}(x)\left(\sum_{n=0}^{\infty} \lambda^{n}(x) p\left(T^{n}(x)+\vartheta_{n}\right)-p\left(x+\vartheta_{0}\right)\right)\right) \\
& =\left(T(x), \sum_{n=1}^{\infty} \lambda(T(x)) \cdots \lambda\left(T^{n-1}(x)\right) p\left(T^{n}(x)+\vartheta_{n}\right)\right) \\
& =\left(T(x), w_{\tau(\theta)}(T(x))\right) .
\end{aligned}
$$

\section{$\S 2.5$ Statement of results}

We assume that $\hat{T}$ is partially hyperbolic, i.e. there exists $\rho>1$ such that

$$
1<\rho \leq \inf _{x \in S^{1}} \lambda(x) \inf _{x \in S^{1}} T^{\prime}(x) .
$$


That is $\hat{T}$ is partially hyperbolic if the maximum rate of exponential expansion in the $\mathbb{R}$-direction is strictly less than the maximum rate of exponential expansion in the $S^{1}$-direction. In the case where $\lambda$ is constant and $T(x)=b x \bmod 1$, this reduces to $\lambda b>1$.

There is an obvious obstruction to the regularity of $w$ : if there exists a smooth solution $u$ to the cohomological equation $p(x)=\lambda(x) u(T(x))-u(x)$ then $w=u$ and the graph of $w$ is as smooth as $u$. Generically this does not happen [HNW].

The following gives an upper bound for $\operatorname{dim}_{H}\left(\operatorname{graph}\left(w_{\theta}\right)\right)$; note that in this case the bound holds for all $\theta \in \Omega$.

\section{Proposition 2.2 (Upper bound)}

Suppose that $T: S^{1} \rightarrow S^{1}$ is a $C^{1+\varepsilon}$ uniformly expanding map of the circle. Let $\lambda: S^{1} \rightarrow(0,1)$ be $C^{1}$. Let $p: S^{1} \rightarrow \mathbb{R}$ be $C^{1}$. Suppose that the partial hyperbolicity assumption (10) holds. Then there exists a unique $s>0$ such that

$$
P\left((1-s) \log T^{\prime}+\log \lambda\right)=0 .
$$

Moreover $\operatorname{dim}_{H}\left(\operatorname{graph}\left(w_{\theta}\right)\right) \leq s$ for every $\theta \in \Omega$.

For the lower bound we need some additional smoothness assumptions on $p$. Recall that $p(\vartheta)$ has a critical point of order $k$ if $p^{\prime}(\vartheta)=p^{\prime \prime}(\vartheta)=$ $\cdots=p^{(k-1)}(\vartheta)=0$ but $p^{(k)}(\vartheta) \neq 0$. We say that a smooth function $p$ satisfies the critical point hypothesis if there exists $r>0$ such that for all $a \in(0,1)$ and $c \in \mathbb{R}$, the critical points of $p(a+\vartheta)-c p(\vartheta)$ have orders strictly less than $r$. This assumption is satisfied by any polynomial, any finite sum of trigonometric functions, and we would expect it to hold generically for smooth functions on $S^{1}$.

\section{Proposition 2.3 (Lower bound)}

Suppose that $T: S^{1} \rightarrow S^{1}$ is a $C^{1+\varepsilon}$ uniformly expanding map of the circle. Let $\lambda: S^{1} \rightarrow(0,1)$ be such that $\log \lambda$ is Hölder continuous. Let $p: S^{1} \rightarrow \mathbb{R}$ satisfy the critical point hypothesis. Suppose that the partial hyperbolicity assumption (10) holds.

Let $g: S^{1} \rightarrow \mathbb{R}$ be Hölder continuous. Then there exists a unique solution $s_{g}>0$ to

$$
P\left(\left(s_{g}-1\right) \log T^{\prime}+2(g-P(g))-\log \lambda\right)=0 .
$$

Moreover $s_{g} \leq \operatorname{dim}_{H}\left(\operatorname{graph}\left(w_{\theta}\right)\right)$ for almost every $\theta \in \Omega$.

We can now state the main result.

\section{Theorem 2.4}

Suppose that $T: S^{1} \rightarrow S^{1}$ is a $C^{1+\varepsilon}$ uniformly expanding map of the circle. Let $\lambda: S^{1} \rightarrow(0,1)$ be $C^{1}$. Let $p: S^{1} \rightarrow \mathbb{R}$ satisfy the critical point hypothesis. Suppose that the partial hyperbolicity assumption (10) holds. 
Let $s_{0}$ be the unique solution to (11) and let $\mu_{0}$ be the unique equilibrium state of the potential $g_{0}=\left(1-s_{0}\right) \log T^{\prime}+\log \lambda$. Then for almost every $\theta \in \Omega$

$$
\operatorname{dim}_{H}\left(\operatorname{graph}\left(w_{\theta}\right)\right)=1+\frac{h_{\mu_{0}}(T)+\int \log \lambda d \mu_{0}}{\int \log T^{\prime} d \mu_{0}} .
$$

Remark. For an invariant probability measure $\mu$ and continuous function $g: S^{1} \rightarrow \mathbb{R}$, the measure-theoretic pressure of $g$ with respect to $\mu$ is defined to be $P_{\mu}(g)=h_{\mu}(T)+\int g d \mu$ and can be regarded as a generalisation of entropy. Thus we can regard the right-hand side of (13) as the sum of the dimension of $\operatorname{graph}\left(w_{\theta}\right)$ in the $S^{1}$-direction and the dimension of $\operatorname{graph}\left(w_{\theta}\right)$ in the $\mathbb{R}$-direction, where the latter has the form of (a generalisation of) entropy divided by the Lyapunov exponent of $T$ with respect to $\mu_{0}$.

Remark. If $T(x)=b x \bmod 1$ where $b \geq 2$ is an integer, then $\mu_{0}$ is the equilibrium state of $\log \lambda$. In this case, (13) takes the form

$$
\operatorname{dim}_{H}\left(\operatorname{graph}\left(w_{\theta}\right)\right)=1+\frac{P(\log \lambda)}{\log b} \text { a.e.. }
$$

If in addition $\lambda(x)=\lambda$ is constant then $\mu_{0}$ is Lebesgue measure, $h_{\mu_{0}}(T)=$ $\log b$ and we rederive the result in $[\mathrm{H}]$.

Proof of Theorem 2.4. Let $s_{0}, g_{0}$ and $\mu_{0}$ be as in the statement of the theorem. Note that $P\left(g_{0}\right)=0$. By the variational principle, we have that

$$
h_{\mu_{0}}(T)+\left(1-s_{0}\right) \int \log T^{\prime} d \mu_{0}+\int \log \lambda d \mu_{0}=0 .
$$

It follows that

$$
s_{0}=1+\frac{h_{\mu_{0}}(T)+\int \log \lambda d \mu_{0}}{\int \log T^{\prime} d \mu_{0}}
$$

and from Proposition 2.2 that $\operatorname{dim}_{H}\left(\operatorname{graph}\left(w_{\theta}\right)\right) \leq s_{0}$ for almost every $\theta \in \Omega$.

Let $s_{1}$ be the unique solution to $(12)$ with potential $g_{0}$. It follows from Proposition 2.3 that $s_{1} \leq \operatorname{dim}_{H}\left(\operatorname{graph}\left(w_{\theta}\right)\right)$ for almost every $\theta \in \Omega$. It remains to show that $s_{1}=s_{0}$. First note that from (12) we have that

$$
P\left(\left(s_{1}-1\right) \log T^{\prime}-\log \lambda+2\left(1-s_{0}\right) \log T^{\prime}+2 \log \lambda\right)=0,
$$

that is

$$
P\left(\left(1-2 s_{0}+s_{1}\right) \log T^{\prime}+\log \lambda\right)=0 .
$$

As $s_{0}$ is the unique solution to $P\left(\left(1-s_{0}\right) \log T^{\prime}+\log \lambda\right)=0$, it follows that $1-s_{0}=1-2 s_{0}+s_{1}$, i.e. $s_{0}=s_{1}$, and the result follows. 


\section{$\S 3 \quad$ Proof of Proposition 2.2}

The upper bound continues to be true without the addition of the random phase shift $\theta$. Indeed, [Be] considers invariant graphs that include those of the form $w(x)=\sum_{n=0}^{\infty} \lambda(x) \lambda(T(x)) \cdots \lambda\left(T^{n-1}(x)\right) p\left(T^{n}(x)\right)$ and proves that the box dimension of $\operatorname{graph}(w)$ is given by (11). The following proposition in a non-random context is proved in [Be]. For a function $\gamma: S^{1} \rightarrow \mathbb{R}$ and an interval $I \subset[0,1]$, the height of $\gamma$ over $I$, denoted by height ${ }_{I}(\gamma)$, is defined to be

$$
\operatorname{height}_{I}(\gamma)=\sup _{s, t \in I}|\gamma(s)-\gamma(t)|
$$

\section{Proposition 3.1}

Let $\left[x_{0}, x_{1}, \ldots, x_{n-1}\right] \in \mathcal{C}_{n}$ be a cylinder of rank $n$. Then there exists $C>0$ (independent of $x, n$ ) such that

$$
\operatorname{height}_{\left[x_{0}, x_{1}, \ldots, x_{n-1}\right]}\left(w_{\theta}\right) \leq C \lambda^{n}(x) .
$$

Proof. Let $T_{i}$ be the inverse branches of $T$. For each $\vartheta \in S^{1}$ define

$$
\hat{T}_{i, \vartheta}(x, y, \theta)=\left(T_{i}(x), \lambda\left(T_{i}(x)\right) y+p\left(T_{i}(x)+\vartheta\right), \vartheta \theta\right)
$$

where if $\theta=\left(\vartheta_{0}, \vartheta_{1}, \ldots\right)$ then $\vartheta \theta$ denotes the sequence $\left(\vartheta, \vartheta_{0}, \vartheta_{1}, \ldots\right)$. Then $\hat{T} \hat{T}_{i, \vartheta}(x, y, \theta)=(x, y, \theta)$ so that $\hat{T}_{i, \vartheta}$ are the inverse branches of $\hat{T}$.

Consider the action of $\hat{T}_{i, \vartheta}$ on the first two coordinates. This has derivative

$$
\left(\begin{array}{cc}
T_{i}^{\prime}(x) & 0 \\
S_{i, \vartheta}(x, y) & \lambda\left(T_{i}(x)\right)
\end{array}\right)
$$

where $S_{i, \vartheta}(x, y)=\lambda^{\prime}\left(T_{i}(x)\right) T_{i}^{\prime}(x) y+p^{\prime}\left(T_{i}(x)+\vartheta\right) T_{i}^{\prime}(x)$.

First note that

$$
\begin{aligned}
& \left(\hat{T}_{x_{0}, \vartheta_{0}} \circ \hat{T}_{x_{1}, \vartheta_{1}}\right)^{\prime}(x, y) \\
& \quad=\hat{T}_{x_{0}, \vartheta_{0}}^{\prime}\left(\hat{T}_{x_{1}, \vartheta_{1}}(x, y)\right) \hat{T}_{x_{1}, \vartheta_{1}}^{\prime}(x, y) \\
& \quad=\left(\begin{array}{cc}
T_{x_{0}}^{\prime}\left(T_{x_{1}}(x)\right) & 0 \\
S_{x_{0}, \vartheta_{0}}\left(\hat{T}_{x_{1}, \vartheta_{1}}(x, y)\right) & \lambda\left(T_{x_{0}} T_{x_{1}}(x)\right)
\end{array}\right)\left(\begin{array}{cc}
T_{x_{1}}^{\prime}(x) & 0 \\
S_{x_{1}, \vartheta_{1}}(x, y) & \lambda\left(T_{x_{1}}(x)\right)
\end{array}\right) \\
& =\left(\begin{array}{cc}
T_{x_{0}}^{\prime}\left(T_{x_{1}}(x)\right) T_{x_{1}}^{\prime}(x) & 0 \\
S_{x_{0}, \vartheta_{0}}\left(\hat{T}_{x_{1}}(x, y)\right) T_{x_{1}}^{\prime}(x)+\lambda\left(T_{x_{0}} T_{x_{1}}(x)\right) S_{x_{1}, \vartheta_{1}}(x, y) & \lambda\left(T_{x_{0}} T_{x_{1}}(x)\right) \lambda\left(T_{x_{1}}(x)\right)
\end{array}\right) .
\end{aligned}
$$

Induction then allows us to write the derivative of $\left(\hat{T}_{x_{0}, \vartheta_{0}} \hat{T}_{x_{1}, \vartheta_{1}} \cdots \hat{T}_{x_{n-1}, \vartheta_{n-1}}\right)(x, y)$ in the form

$$
\left(\begin{array}{cc}
\prod_{j=0}^{n-1} T_{x_{j}}^{\prime}\left(T_{x_{j+1}} \cdots T_{x_{n-1}}(x)\right) & 0 \\
(*) & \prod_{j=0}^{n-1} \lambda\left(T_{x_{j}} \cdots T_{x_{n-1}}(x)\right)
\end{array}\right)
$$


where

$$
\begin{aligned}
(*)= & \sum_{k=0}^{n-1} \prod_{j=0}^{k-1} \lambda\left(T_{x_{j}} \cdots T_{x_{n-1}}(x)\right) S_{x_{k}, \vartheta_{k}}\left(\hat{T}_{x_{k+1}, \vartheta_{k+1}} \cdots \hat{T}_{x_{n-1}, \vartheta_{n-1}}(x, y)\right) \\
& \times \prod_{j=k+1}^{n-1} T_{x_{j}}^{\prime}\left(T_{x_{j+1}} \cdots T_{x_{n-1}}(x)\right)
\end{aligned}
$$

and products such as $\prod_{n}^{n-1}$, etc, are interpreted as being empty.

Let $J \subset I$ be a subinterval and let $\gamma(t)=\left(\gamma_{H}(t), \gamma_{V}(t)\right)$ be a differentiable curve in $J \times \mathbb{R}$ (we use $H, V$ to denote the 'horizontal' (along $I$ ) and 'vertical' (along $\mathbb{R}$ ) directions, respectively, and write $\pi_{H}, \pi_{V}$ to denote the corresponding projections). Choose points $x^{+}, x^{-} \in\left[x_{0}, x_{1}, \ldots, x_{n-1}\right]$ such that

$$
\operatorname{height}_{\left[x_{0}, x_{1}, \ldots, x_{n-1}\right]}\left(w_{\theta}\right)=w_{\theta}\left(x^{+}\right)-w_{\theta}\left(x^{-}\right) .
$$

Let $\gamma_{0}$ denote the straight-line segment joining $\left(x^{+}, w_{\theta}\left(x^{+}\right)\right)$to $\left(x^{-}, w_{\theta}\left(x^{-}\right)\right)$ and let $\gamma=\hat{T} \gamma_{0}$. The vertical height of $w_{\theta}$ over $\left[x_{0}, \ldots, x_{n-1}\right]$ is then bounded by

$$
\begin{aligned}
& \text { height }_{\left[x_{0}, x_{1}, \ldots, x_{n-1}\right]}\left(\hat{T}_{x_{0}, \vartheta_{0}} \hat{T}_{x_{1}, \vartheta_{1}} \cdots \hat{T}_{x_{n-1}, \vartheta_{n-1}} \gamma\right) \\
& \leq \int\left|\pi_{V}\left(\hat{T}_{x_{0}, \vartheta_{0}} \hat{T}_{x_{1}, \vartheta_{1}} \cdots \hat{T}_{x_{n-1}, \vartheta_{n-1}} \gamma\right)^{\prime}(t)\right| d t \\
& \leq \int \mid \sum_{k=0}^{n-1} \prod_{j=0}^{k-1} \lambda\left(T_{x_{j}} \cdots T_{x_{n-1}} \gamma_{H}(t)\right) S_{x_{k}, \vartheta_{k}}\left(\hat{T}_{x_{k+1}, \vartheta_{k+1}} \cdots \hat{T}_{x_{n-1}, \vartheta_{n-1}}(\gamma(t))\right) \\
& \quad \times \prod_{j=k+1}^{n-1} T_{x_{j}}^{\prime}\left(T_{x_{j+1}} \cdots T_{x_{n-1}}\left(\gamma_{H}(t)\right)\right)|| \gamma_{H}^{\prime}(t) \mid d t \\
& \quad+\int\left|\prod_{j=0}^{n-1} \lambda\left(T_{x_{j}} \cdots T_{x_{n-1}}\left(\gamma_{H}(t)\right)\right)\right|\left|\gamma_{V}^{\prime}(t)\right| d t .
\end{aligned}
$$

By the partial hyperbolicity assumption (10), we have that

$$
\sup _{x, i} T_{i}^{\prime}(x) \leq \rho^{-1} \lambda(x)
$$

where $0<\rho^{-1}<1$.

Note that $\left|w_{\theta}(x)\right| \leq|p|_{\infty} /\left(1-|\lambda|_{\infty}\right)$. Let $A=\sup _{i, \vartheta} \sup _{x, y}\left|S_{i, \vartheta}(x)\right|<\infty$ where the supremum over $x, y$ is taken over $x \in I,|y| \leq|p|_{\infty} /\left(1-|\lambda|_{\infty}\right)$. Then we can bound the first integral in (14) by

$$
A \sum_{k=0}^{n-1} \prod_{j=0}^{k-1} \lambda\left(T_{x_{j}} \cdots T_{x_{n-1}}\left(\gamma_{H}(t)\right)\right) \prod_{j=k+1}^{n-1} \lambda\left(T_{x_{j+1}} \cdots T_{x_{n-1}}\left(\gamma_{H}(t)\right)\right) \rho^{j-n}\left|\gamma_{H}^{\prime}(t)\right| .
$$


By Lemma 2.1 we can bound this by

$$
\prod_{j=0}^{n-1} \lambda\left(T^{j}(x)\right) \times C \sum_{k=0}^{n-1} \rho^{-k} \leq C^{\prime} \prod_{j=0}^{n-1} \lambda\left(T^{j}(x)\right)
$$

for some constants $C, C^{\prime}>0$. Hence

$$
\begin{aligned}
& \text { height }_{\left[x_{0}, x_{1} \ldots, x_{n-1}\right]}\left(\hat{T}_{x_{0}, \vartheta_{0}} \hat{T}_{x_{1}, \vartheta_{1}} \cdots \hat{T}_{x_{n-1}, \vartheta_{n-1}} \gamma\right) \\
& \quad=\quad w_{\theta}\left(x^{+}\right)-w_{\theta}\left(x^{-}\right) \\
& \leq\left(C^{\prime} \int\left|\gamma_{H}^{\prime}(t)\right|+\left|\gamma_{V}^{\prime}(t)\right| d t\right) \prod_{j=0}^{n-1} \lambda\left(T^{j}(x)\right) \\
& \leq\left(C^{\prime}+\left|w_{\theta}\right|_{\infty}\right) \prod_{j=0}^{n-1} \lambda\left(T^{j}(x)\right)
\end{aligned}
$$

and the result follows.

Proof of Proposition 2.2. First note that $(1-s) \log T^{\prime}+\log \lambda \leq-s \log \beta+$ $\left\|\log T^{\prime}+\log \lambda\right\|_{\infty}$. Hence

$$
P\left((1-s) \log T^{\prime}+\log \lambda\right) \leq-s \log \beta+\left\|\log T^{\prime}+\log \lambda\right\|_{\infty}+h_{\text {top }}(T)
$$

where $h_{\text {top }}(T)=P(0)$ is the topological entropy of $T$. As $\log \beta>0$, it follows that $P\left((1-s) \log T^{\prime}+\log \lambda\right) \rightarrow-\infty$ as $s \rightarrow \infty$. By partial hyperbolicity, $\log T^{\prime}+\log \lambda \geq \log \rho>0$ so that when $s=0, P\left((1-s) \log T^{\prime}+\log \lambda\right) \geq$ $P(\log \rho)>0$. As the pressure depends continuously on $s$, it follows that there is a unique value $s>0$ that solves (11).

Let $s_{0}$ be the unique solution to $P\left(\left(1-s_{0}\right) \log T^{\prime}+\log \lambda\right)$. Let $s>s_{0}$. Then $P\left((1-s) \log T^{\prime}+\log \lambda\right)=-\eta<0$. By the partial hyperbolicity hypothesis, $\log \lambda+\log T^{\prime}>0$, hence $P\left(-s \log T^{\prime}\right) \leq-\eta<0$. Hence there exists $N$ such that if $n \geq N$ then

$$
\sum_{\left[x_{0}, x_{1}, \ldots, x_{n-1}\right] \in \mathcal{C}_{n}} \prod_{j=0}^{n-1} T^{\prime}\left(T^{j}(x)\right)^{1-s} \prod_{j=0}^{n-1} \lambda\left(T^{j}(x)\right)<e^{\frac{-n \eta}{2}}
$$

and

$$
\sum_{\left[x_{0}, x_{1}, \ldots, x_{n-1}\right] \in \mathcal{C}_{n}} \prod_{j=0}^{n-1} T^{\prime}\left(T^{j}(x)\right)^{-s}<e^{\frac{-n \eta}{2}}
$$

Let $\delta>0$. Then there exists $N$ such that if $n \geq N$ then $\operatorname{diam}\left[x_{0}, x_{1}, \ldots, x_{n-1}\right]<$ $\delta$ for all cylinders of rank $n$. By the Mean Value Theorem, for each $\left[x_{0}, x_{1}, \ldots, x_{n-1}\right] \in$ 
$\mathcal{C}_{n}$, choose $x \in\left[x_{0}, x_{1}, \ldots, x_{n-1}\right]$ such that

$$
\operatorname{diam}\left[x_{0}, x_{1}, \ldots, x_{n-1}\right]=\prod_{j=0}^{n-1} T^{\prime}\left(T^{j}(x)\right)^{-1} .
$$

Consider the graph of $w_{\theta}$ over the cylinder $\left[x_{0}, x_{1}, \ldots, x_{n-1}\right]$. This has height at most $C \lambda^{n}(x)$. Hence at most

$$
\frac{C \lambda^{n}(x)}{\operatorname{diam}\left[x_{0}, x_{1}, \ldots, x_{n-1}\right]}+1=C\left(\prod_{j=0}^{n-1} T^{\prime}\left(T^{j}(x)\right) \prod_{j=0}^{n-1} \lambda\left(T^{j}(x)\right)\right)+1
$$

sets of diameter at most $\operatorname{diam}\left[x_{0}, x_{1}, \ldots, x_{n-1}\right]$ are needed to cover the graph of $w_{\theta}$ over $\left[x_{0}, x_{1}, \ldots, x_{n-1}\right]$. Taking all such sets over all cylinders of rank $n$ gives an open cover $\mathcal{U}_{n}$ of $\operatorname{graph}\left(w_{\theta}\right)$ of diameter at most $\delta$. Hence

$$
\begin{aligned}
& \mathcal{H}_{\delta}^{s}\left(\operatorname{graph}\left(w_{\theta}\right)\right) \\
& \leq \sum_{U \in \mathcal{U}_{n}}(\operatorname{diam} U)^{s} \\
& \leq \sum_{\left[x_{0}, x_{1}, \ldots, x_{n-1}\right] \in \mathcal{C}_{n}}\left(C \prod_{j=0}^{n-1} T^{\prime}\left(T^{j}(x)\right) \prod_{j=0}^{n-1} \lambda\left(T^{j}(x)\right)+1\right)(\operatorname{diam} U)^{s} \\
& \leq \quad \sum_{\left[x_{0}, x_{1}, \ldots, x_{n-1}\right] \in \mathcal{C}_{n}} \prod_{j=0}^{n-1} T^{\prime}\left(T^{j}(x)\right)^{1-s} \prod_{j=0}^{n-1} \lambda\left(T^{j}(x)\right) \\
& \quad+\sum_{\left[x_{0}, x_{1}, \ldots, x_{n-1}\right] \in \mathcal{C}_{n}} \prod_{j=0}^{n-1} T^{\prime}\left(T^{j}(x)\right)^{-s} \\
& \leq(C+1) e^{\frac{-n \eta}{2}} .
\end{aligned}
$$

Letting $n \rightarrow \infty$ we have that $\mathcal{H}_{\delta}^{s}\left(\operatorname{graph}\left(w_{\theta}\right)\right)=0$. Letting $\delta \rightarrow 0$, we have that $\mathcal{H}^{s}\left(\operatorname{graph}\left(w_{\theta}\right)\right)=0$. Hence $\operatorname{dim}_{H} \operatorname{graph}\left(w_{\theta}\right) \leq s$. As $s>s_{0}$ is arbitrary, the result follows.

\section{$\S 4 \quad$ Proof of Proposition 2.3}

We first need the following bounded distortion estimate on Bowen balls.

\section{Lemma 4.1}

Let $f: S^{1} \rightarrow \mathbb{R}$ be Hölder continuous of exponent $\alpha$. Let $\delta$ be less than the injectivity radius of $T$. Then there exists a constant $C>0$ such that for all balls $B_{n, \delta}(z)$ and all $x, y \in B_{n, \delta}(z)$ we have

$$
\left|\sum_{j=0}^{n-1} f\left(T^{j}(x)\right)-f\left(T^{j}(y)\right)\right| \leq C|f|_{\alpha} \delta^{\alpha}
$$


Proof. To see this note that if $x, y, \delta$ are as in the statement of the lemma, then $d(x, y) \leq \sup _{x \in S^{1}}\left(T^{-1}\right)^{\prime}(x) d(x, y) \leq \beta^{-1} d(x, y)$. Inductively we obtain that

$$
\begin{aligned}
\left|\sum_{j=0}^{n-1} f\left(T^{j}(x)\right)-f\left(T^{j}(y)\right)\right| & \leq|f|_{\alpha} \sum_{j=0}^{n-1} \frac{\delta^{\alpha}}{\beta^{j \alpha}} \\
& \leq \frac{|f|_{\alpha}}{1-\beta^{-\alpha}} \delta^{\alpha} .
\end{aligned}
$$

Remark. As $\lambda$ is $C^{1}, \log \lambda$ is Hölder continuous. It follows immediately from Lemma 4.1 that, if $\delta>0$ is less than the injectivity radius of $T$, then there exists $C_{\lambda}>0$ such that for all balls $B_{n, \delta}(z)$ and all $x, y \in B_{n, \delta}(z)$ we have

$$
\frac{1}{C_{\lambda}} \leq \frac{\lambda^{n}(x)}{\lambda^{n}(y)} \leq C_{\lambda}
$$

Proof of Proposition 2.3. First note that

$(s-1) \log T^{\prime}+2(g-P(g))-\log \lambda \geq s \log \beta+2(g-P(g))-\left\|\log T^{\prime}+\log \lambda\right\|_{\infty}$.

Hence $P\left((s-1) \log T^{\prime}+2(g-P(g))-\log \lambda\right) \geq s \log \beta+P(2(g-P(g)))-$ $\left\|\log T^{\prime}+\log \lambda\right\|_{\infty}$ so that $P\left((s-1) \log T^{\prime}+2(g-P(g))-\log \lambda\right) \rightarrow \infty$ as $s \rightarrow \infty$. Note that $-\log T^{\prime}+2(g-P(g))-\log \lambda \leq-\log \rho+2(g-P(g))$. Hence when $s=0$,

$$
\begin{aligned}
& P\left((s-1) \log T^{\prime}+2(g-P(g))-\log \lambda\right) \\
& \quad=P\left(-\log T^{\prime}+2(g-P(g))-\log \lambda\right) \\
& \quad \leq-\log \rho+P(2 g)-2 P(g) .
\end{aligned}
$$

As $P(2 g)-2 P(g)<0$, we see that $P\left((s-1) \log T^{\prime}+2(g-P(g))-\log \lambda\right)<0$ when $s=0$. By the continuity of pressure, there exists a unique value $s_{g}>0$ solving (12).

Let $g: S^{1} \rightarrow \mathbb{R}$ be Hölder continuous and let $\mu_{g}$ be the associated equilibrium state. Let $\theta=\left(\vartheta_{j}\right)_{j=0}^{\infty} \in \Omega$. Define a measure $\hat{\mu}_{g}$ on $S^{1} \times \mathbb{R}$ $\operatorname{supported}$ on $\operatorname{graph}\left(w_{\theta}\right)$ by $\hat{\mu}_{g}(E)=\mu_{g}\left\{x \in S^{1} \mid\left(x, w_{\theta}(x)\right) \in E\right\}$. We want to show that if $s<s_{g}$ where $s_{g}$ is determined by (12) then

$$
I_{s}\left(\hat{\mu}_{g}\right)=\iint_{S^{1} \times S^{1}} \frac{d \mu_{g}(x) d \mu_{g}(y)}{\left((x-y)^{2}+\left(w_{\theta}(x)-w_{\theta}(y)\right)^{2}\right)^{s / 2}}<\infty
$$

for $d \theta$-almost every $\theta \in \Omega$. To do this, it is sufficient to assume that $s>1$ and prove that

$$
E_{s}=\int_{\Omega} I_{s}\left(\hat{\mu}_{g}\right) d \theta<\infty
$$


By Fubini's theorem we can write

$$
E_{s}=\iint_{S^{1} \times S^{1}} \int_{\Omega} \frac{d \theta d \mu_{g}(x) d \mu_{g}(y)}{\left((x-y)^{2}+\left(w_{\theta}(x)-w_{\theta}(y)\right)^{2}\right)^{s / 2}} .
$$

Let $r>0$ be determined by the critical point hypothesis. Choose $\delta<$ $1 / 4 \beta$ and shrink $\delta$ further, if necessary, so that $\left\|T^{\prime}\right\|_{\infty}^{r} \delta$ is less than the injectivity radius of $T$. Note that $\delta$ is an expansivity constant for $T$. Let

$$
X_{n}^{r}=\left\{(x, y) \in S^{1} \times S^{1} \mid d_{n}(x, y)<\delta, \delta \leq d\left(T^{n}(x), T^{n}(y)\right)\right\} .
$$

Then clearly $\bigcup_{n=0}^{\infty} X_{n}^{r} \subset\left\{(x, y) \in S^{1} \times S^{1} \mid d(x, y)<\delta\right\}=\Delta_{\delta}$, a neighbourhood of the diagonal in $S^{1} \times S^{1}$.

Note that if $(x, y) \in\left(S^{1} \times S^{1}\right) \backslash \Delta_{\delta}$ then $|x-y| \geq \delta$. Hence

$$
\begin{aligned}
& \iint_{\left(S^{1} \times S^{1}\right) \backslash \Delta_{\delta}} \int_{\Omega} \frac{d \theta d \mu_{g}(x) d \mu_{g}(y)}{\left((x-y)^{2}+\left(w_{\theta}(x)-w_{\theta}(y)\right)^{2}\right)^{s / 2}} \\
& \quad \leq \iint_{\left(S^{1} \times S^{1}\right) \backslash \Delta_{\delta}} \int_{\Omega} \frac{d \theta d \mu_{g}(x) d \mu_{g}(y)}{|x-y|^{s}} \\
& \leq \frac{1}{\delta^{s}} .
\end{aligned}
$$

Hence

$$
E_{s}=\frac{1}{\delta^{s}}+\iint_{\Delta_{\delta}} \int_{\Omega} \frac{d \theta d \mu_{g}(x) d \mu_{g}(y)}{\left((x-y)^{2}+\left(w_{\theta}(x)-w_{\theta}(y)\right)^{2}\right)^{s / 2}}
$$

and it remains to show that the second term in (18) is finite.

Fix $x, y \in X_{n}^{r}$. Let $z_{x, y}(\theta)=w_{\theta}(x)-w_{\theta}(y)$ and let $h_{x, y}$ denote the density of $z_{x, y}$. Then the second term in (18) can be written as

$$
E_{s}(\delta)=\sum_{n=0}^{\infty} \iint_{X_{n}^{r}} \int_{-\infty}^{\infty} \frac{h_{x, y}\left(z_{x, y}\right) d z_{x, y} d \mu_{g}(x) d \mu_{g}(y)}{\left((x-y)^{2}+z_{x, y}^{2}\right)^{s / 2}} .
$$

Let $z_{x, y}=|x-y| u_{x, y}$ so that $d z_{x, y}=|x-y| d u_{x, y}$. Then

$$
E_{s}(\delta)=\sum_{n=0}^{\infty} \iint_{X_{n}^{r}} \int_{-\infty}^{\infty} \frac{|x-y|^{1-s} h_{x, y}\left(|x-y| u_{x, y}\right) d u_{x, y} d \mu_{g}(x) d \mu_{g}(y)}{\left(1+u_{x, y}^{2}\right)^{s / 2}} .
$$

Let

$$
K(s)=\int_{-\infty}^{\infty} \frac{d u}{\left(1+u^{2}\right)^{s / 2}}
$$

and note that $K(s)<\infty$ if $s>1$. Then

$$
E_{s}(\delta) \leq K(s) \sum_{n=0}^{\infty} \iint_{X_{n}^{r}}|x-y|^{1-s} \sup _{u \in \mathbb{R}} h_{x, y}(u) d \mu_{g}(x) d \mu_{g}(y) .
$$


Let $F_{n}$ be an $(n, \delta)$-spanning set which achieves the infimum in (6) for the potential $(1-s) \log T^{\prime}+\log \lambda$. For $z \in F_{n}$ let

$$
X_{n}^{r}(z)=X_{n}^{r} \cap\left(B_{n, 2 \delta}(z) \times B_{n, 2 \delta}(z)\right) .
$$

Let $(x, y) \in X_{n}^{r}$. As $F_{n}$ is $(n, \delta)$-spanning, there exists $z \in F_{n}$ such that $d_{n}(x, z)<\delta$. Hence $d_{n}(y, z) \leq d(y, x)+d(x, z) \leq 2 \delta$. Clearly $d_{n}(x, z)<2 \delta$, so it follows that $(x, y) \in X_{n}^{r}(z)$. Hence

$$
X_{n}^{r}=\bigcup_{z \in F_{n}} X_{n}^{r}(z)
$$

Hence

$$
E_{s}(\delta) \leq K(s) \sum_{n=0}^{\infty} \sum_{z \in F_{n}} \iint_{X_{n}^{r}(z)}|x-y|^{1-s} \sup _{u \in \mathbb{R}} h_{x, y}(u) d \mu_{g}(x) d \mu_{g}(y) .
$$

We first bound $h_{x, y}$.

\section{Lemma 4.2}

Let $x, y \in B_{n, 2 \delta}(z)$. Then $\sup _{u \in \mathbb{R}} h_{x, y}(u)<C_{h} \lambda^{n}(z)^{-1}$ where $C_{h}>0$ is a constant independent of $x, y, n$.

Proof. Write

$$
\begin{aligned}
z_{x, y}(\theta) & =w_{\theta}(x)-w_{\theta}(y) \\
& =\sum_{k=0}^{\infty} \lambda^{k}(x) p\left(T^{k}(x)+\vartheta_{k}\right)-\lambda^{k}(y) p\left(T^{k}(y)+\vartheta_{k}\right) \\
& =\sum_{k=0}^{\infty} z_{k, x, y}\left(\vartheta_{k}\right) .
\end{aligned}
$$

Let $h_{k, x, y}$ denote the density of $z_{k, x, y}$. As the $\vartheta_{k}$ s are independent, the density of $z_{x, y}$ is the convolution of the densities of the $z_{k, x, y}$. Hence

$$
h_{x, y}=\stackrel{\infty}{\underset{k}{*}} h_{k, x, y}
$$

Now a bound on the convolution of the $h_{k, x, y}$ for finitely many values of $n$ will automatically be a bound on the infinite convolution in (19). Hence

$$
h_{x, y} \leq \stackrel{r-1}{*} h_{j=0} h_{n+j, x, y}
$$

where $n$ is chosen so that $x, y$ are in the same Bowen ball $B_{n, 2 \delta}(z)$. By Hölder's inequality we can bound

$$
\underset{j=0}{\stackrel{r-1}{*}} h_{n+j, x, y} \leq\left\|h_{n, x, y} * \cdots * h_{n+r-2, x, y}\right\|_{r}\left\|h_{n+r-1, x, y}\right\|_{\frac{r}{r-1}} .
$$


Repeated applications of Young's inequality then implies that

$$
\left\|h_{n, x, y} * \cdots * h_{n+r-2, x, y}\right\|_{r} \leq\left\|h_{n, x, y}\right\|_{\frac{r}{r-1}} \cdots\left\|h_{n+r-2, x, y}\right\|_{\frac{r}{r-1}} .
$$

Hence

$$
h_{x, y} \leq \prod_{j=0}^{r-1}\left\|h_{n+j, x, y}\right\|_{\frac{r}{r-1}} .
$$

Define $z_{i, x, y}^{\prime}\left(\vartheta_{i}\right)$ by

$$
\begin{aligned}
z_{i, x, y}\left(\vartheta_{i}\right) & =\lambda^{i}(x)\left(p\left(T^{i}(x)+\vartheta_{i}\right)-\frac{\lambda^{i}(y)}{\lambda^{i}(x)} p\left(T^{i}(y)+\vartheta_{i}\right)\right) \\
& =\lambda^{i}(x) z_{i, x, y}^{\prime}\left(\vartheta_{i}\right) .
\end{aligned}
$$

Let $h_{i, x, y}^{\prime}$ denote the density of $z_{i, x, y}^{\prime}\left(\vartheta_{i}\right)$. Then

$$
h_{i, x, y}(u)=\frac{1}{\lambda^{i}(x)} h_{i, x, y}^{\prime}\left(\frac{u}{\lambda^{i}(x)}\right) .
$$

We will prove that

$$
\left\|h_{n+j, x, y}^{\prime}\right\|_{\frac{r}{r-1}} \leq M
$$

for $j=0, \ldots, r-1$, for some $M$ independent of $x, y$. It then follows from (21) that

$$
\left\|h_{n+j, x, y}\right\|_{\frac{r}{r-1}} \leq M \lambda^{n+j}(x)^{-1 / r} \leq\|\lambda\|_{\infty}^{-j / r} M \lambda^{n}(x)^{-1 / r} .
$$

Hence from (20)

$$
h_{x, y} \leq M^{r}\|\lambda\|_{\infty}^{(r-1) / 2} \lambda^{n}(x)^{-1} \leq C_{h} \lambda^{n}(z)^{-1} .
$$

where the last equality follows from the remark following Lemma 4.1.

It remains to prove $(22)$. Write $\vartheta_{j}^{\prime}=T^{j}(y)+\vartheta_{j}$ so that

$$
z_{n+j, x, y}^{\prime}\left(\vartheta_{n+j}\right)=p\left(T^{n+j}(x)-T^{n+j}(y)+\vartheta_{n+j}^{\prime}\right)-\frac{\lambda^{n+j}(y)}{\lambda^{n+j}(x)} p\left(\vartheta_{n+j}^{\prime}\right) .
$$

Now $x, y \in X_{n}^{r}(z)$. Hence $d\left(T^{j}(x), T^{j}(y)\right) \leq \delta$ for $0 \leq j \leq n-1$ and $\delta \leq$ $d\left(T^{n}(x), T^{n}(y)\right)$. Recalling that $\beta \leq \inf _{x \in S^{1}} T^{\prime}(x)$ it follows that $\beta^{j-1} \delta \leq$ $d\left(T^{n+j}(x), T^{n+j}(y)\right)<\left\|T^{\prime}\right\|_{\infty}^{j} \delta$ for $j=0,1, \ldots, r-1$. In particular, there exists $\kappa>0$ such that

$$
\kappa \leq\left|T^{n+j}(x)-T^{n+j}(y)\right|<1-\kappa
$$

for $j=0,1, \ldots, r-1$.

Let $\Lambda=\sup _{x \in S^{1}} \lambda(x) / \inf _{x \in S^{1}} \lambda(x)$. By (15) there exists $C_{\lambda}>0$ such that

$$
\frac{1}{C_{\lambda} \Lambda^{r}} \leq \frac{\lambda^{n+j}(x)}{\lambda^{n+j}(y)} \leq C_{\lambda} \Lambda^{r}
$$


for $j=0,1, \ldots, r-1$.

Suppose that $q: S^{1} \rightarrow \mathbb{R}$ has a critical point of order $k$ at $x_{0} \in S^{1}$ and $q^{(k)}\left(x_{0}\right)=b$. Then the density of $q$ in a neighbourhood of $q\left(x_{0}\right)$ behaves like $C(b) t^{(1-k) / k}$ where the constant $C(b)$ is of the order $O\left(b^{-1 / k}\right)$. Hence if all the critical points of $q$ have order less than $r$ then the density $h_{q}$ of $q$ is such that $\left\|h_{q}\right\|_{\frac{r}{r-1}}<\infty$. Suppose we have a family $q_{j}: S^{1} \rightarrow \mathbb{R}, j \in J$, which have critical points of order less than $r$. If the values of $q_{j}^{k}\left(x_{0}\right)=b$ where $x_{0}$ is a critical point of order $k$ for $q_{j}$, as $j$ ranges over $J, k<r$, are uniformly bounded away from 0 then there exists $M>0$ such that $\left\|h_{q_{j}}\right\|_{\frac{r}{r-1}} \leq M$ for all $j \in J$.

By the critical point hypothesis, the critical points of

$$
z_{n+j, x, y}^{\prime}\left(\vartheta_{n+j}\right)=p\left(T^{n+j}(x)-T^{n+j}(y)+\vartheta_{n+j}^{\prime}\right)-\frac{\lambda^{n+j}(y)}{\lambda^{n+j}(x)} p\left(\vartheta_{n+j}^{\prime}\right)
$$

have orders less than $r$, and the corresponding $k$ th derivatives $(1 \leq k<r)$ take values bounded away from zero as $\left|T^{n+j}(x)-T^{n+j}(y)\right| \in[\kappa, 1-\kappa]$, $\lambda^{n}(x) / \lambda^{n}(y) \in\left[\left(C_{\lambda} \Lambda^{r}\right)^{-1}, C_{\lambda} \Lambda^{r}\right]$, a compact set. Hence there exists $M>0$ such that $\left\|h_{n+j, x, y}^{\prime}\right\|_{\frac{r}{r-1}} \leq M$ for $j=0, \ldots, r-1$ and all $x, y \in S^{1}$.

To complete the estimate on the bound of $E_{s}$ we need the following result.

\section{Lemma 4.3}

Let $x, y \in B_{n, 2 \delta}(z)$. Then $|x-y|^{1-s} \leq C_{T}\left(T^{n}\right)^{\prime}(z)^{s-1}$ where $C_{T}>0$ is a constant independent of $x, y, n$.

Proof. This follows immediately from Lemma 4.1 and the fact that $s>1$.

From Lemmas 4.2 and 4.3 it follows that

$$
E_{s}(\delta) \leq K(s) C_{h} C_{T} \sum_{n=0}^{\infty} \sum_{z \in F_{n}} \iint_{X_{n}^{r}(z)}\left(T^{n}\right)^{\prime}(z)^{s-1} \lambda^{n}(z)^{-1} d \mu_{g}(x) d \mu_{g}(y)
$$

As the integrand is constant on each ball $B_{n, 2 \delta}(z)$, each $x$ and $y$ in the integrand are in the same ball $B_{n, \delta}(z)$, and $X_{n}^{r}(z) \subset B_{n, 2 \delta}(z) \times B_{n, 2 \delta}(z)$, we can bound $E_{s}(\delta)$ by

$$
\begin{gathered}
K(s) C_{h} C_{T} \sum_{n=0}^{\infty} \sum_{z \in F_{n}}\left(T^{n}\right)^{\prime}(z)^{s-1} \lambda^{n}(z)^{-1} \iint_{B_{n, 2 \delta}(z) \times B_{n, 2 \delta}(z)} d \mu_{g}(x) d \mu_{g}(y) \\
\leq K(s) C_{h} C_{T} \sum_{n=0}^{\infty} \sum_{z \in F_{n}}\left(T^{n}\right)^{\prime}(z)^{s-1} \lambda^{n}(z)^{-1} \mu_{g}\left(B_{n, 2 \delta}(z)\right) \mu_{g}\left(B_{n, 2 \delta}(z)\right) .
\end{gathered}
$$


Hence, by $(8), E_{s}(\delta)$ is bounded above by

$$
\begin{gathered}
K(s) C_{h} C_{T} C(g, 2 \delta)^{2} \sum_{n=0}^{\infty} \sum_{z \in F_{n}} \exp \sum_{j=0}^{n-1}\left((s-1) \log T^{\prime}\left(T^{j}(z)\right)-\log \lambda\left(T^{j}(z)\right)\right. \\
\left.+2\left(g\left(T^{j}(z)\right)-P(g)\right)\right) .
\end{gathered}
$$

As $1<s<s(g)$, where $s(g)$ is the the unique solution to (12), we have that $P\left((s-1) \log T^{\prime}-\log \lambda+2(g-P(g))\right)=-\eta<0$. Then there exists $C>0$ such that for all $n \geq 0$ we have

$$
\begin{aligned}
& \sum_{z \in F_{n}} \exp \sum_{j=0}^{n-1}(s-1) \log T^{\prime}\left(T^{j}(z)\right)-\log \lambda\left(T^{j}(z)\right)+2\left(g\left(T^{j}(z)\right)-P(g)\right) \\
& \quad \leq C \exp ^{\frac{-n \eta}{2}} .
\end{aligned}
$$

In particular, if $1<s<s(g)$ then

$$
E_{s}(\delta) \leq C K(s) C_{h} C_{T} C(g, 2 \delta)^{2} \sum_{n=0}^{\infty} \exp ^{\frac{-n \eta}{2}}<\infty .
$$

By choosing $s$ arbitrarily close to $s(g)$, the result follows.

\section{References}

[Be] T. Bedford, The box dimension of self-affine graphs and repellers, Nonlinearity 2 (1989), 53-71.

[BU] A.S. Besicovitch and H.D. Ursell, Sets of fractional dimensions, V: On dimensional numbers of some continuous curves, J. London Math. Soc. 12 (1937), 18-25.

[HNW] D. Hadjiloucas, M. J. Nicol, and C. P. Walkden, Regularity of invariant graphs over hyperbolic systems, Ergod. Th. \& Dyn. Syst. 22 (2002), 469482.

[H] B. Hunt, The Hausdorff dimension of graphs of Weierstrass functions, Proc. Amer. Math. Soc. 126 (1998), 791-800.

[M] C. McMullen, The Hausdorff dimension of general Sierpinski carpets, Nagoya Math. J. 96 (1984), 19.

[PU] F. Przytycki and M. Urbański, On the Hausdorff dimension of some fractal sets, Studia Math. 93(1989), 155186.

[W] P. Walters, An introduction to ergodic theory, Springer, Berlin, 1982.

Andrew Moss, School of Mathematics, The University of Manchester, Oxford Road, Manchester M13 9PL, U.K., email: amoss@maths.manchester.ac.uk

Charles Walkden, School of Mathematics, The University of Manchester, Oxford Road, Manchester M13 9PL, U.K., email: charles.walkden@manchester.ac.uk 